\title{
PRÁCTICAS DE RECEPCIÓN TELEVISIVA EN ESTUDIANTES DE ENSEÑANZA MEDIA DE PUNTA ARENAS
}

\author{
GUILLERMO MUÑOZ* Y JUAN CARLOS JUDIKIS*
}

\section{RESUMEN}

A pesar de las nuevas tecnologías surgidas en el último tiempo y que se han insertado en las rutinas cotidianas de un amplio sector de la población, la televisión continua perfilándose como uno de los fenómenos culturales mayoritarios. Si bien existen amplios y diversos estudios nacionales e internacionales que indagan en la relación de determinados grupos sociales con el medio televisivo, no existen investigaciones en Punta Arenas que permitan conocer las experiencias específicas de grupos sociales que habitan en este contexto geográfico y sociocultural.

El presente estudio identificó las experiencias de los estudiantes de enseñanza media de Punta Arenas con la televisión desde una perspectiva que no se limitó a la interacción concreta con el aparato televisivo, sino incorporando aspectos vinculados a su rutina cotidiana en el ámbito escolar, familiar y el medio social en general.

De esta forma, utilizando un enfoque cualitativo, se identificó a través de los discursos sociales de estudiantes pertenecientes a diversos colegios de Punta Arenas, diversas prácticas de recepción televisiva que temáticamente coinciden a nivel grupal, a pesar de mantener dinámicas diferentes e irreproducibles a nivel individual. Estas son la estructuración de una rutina cotidiana que determina la interacción con la televisión; la convicción de los estudiantes sobre la influencia del medio; su vinculación con las instituciones sociales; la preferencia de determinados contenidos televisivos; las dinámicas y formas de ver televisión y la existencia de un discurso crítico hacia la programación televisiva.

Así como otras investigaciones han coincidido que la televisión contribuyó a limitar la hegemonía de la escuela como único medio para acceder a la información, también el presente estudio permitió concluir que la institución escolar mantiene su vigencia en las interacciones sociales de los estudiantes. En este contexto, y advirtiendo sobre el significado en las transformaciones de la escuela que pueden o ya están provocando las tecnologías de información, es necesario profundizar y considerar las investigaciones en torno a la interacción de los diversos grupos sociales- niños, adolescentes, adultos, adultos mayores, etc.- con estas visualizaciones no exclusivamente en el contexto escolar, sino en las rutinas cotidianas.

PALABRAS CLAVE: Televisión, recepción, prácticas, estudiantes.

\footnotetext{
Periodista, Universidad de Magallanes. guillermo.munoz@umag.cl.

* Departamento de Educación y Humanidades, Universidad de Magallanes. juan.judikis@umag.cl.
} 


\section{TELEVISION RECEPTION PRACTICES IN HIGH SCHOOL STUDENTS FROM PUNTA ARENAS}

\section{ABSTRACT}

Despite new technologies that have emerged in recent times and that have been inserted in the daily routines of a large segment of the population; television continues emerging as one of the main cultural phenomena. While there are broad and diverse national and international studies that explore the relationship of certain social groups with the television media, there is no research in Punta Arenas that provides insight into the specific experiences of social groups that inhabit this geographical and socio-cultural context.

This study identified the experiences of high school students from Punta Arenas with television from an area which wasn't limited only to the specific interaction with the television apparatus, but including aspects related to their daily routine in schools, family and social environment in general.

Thus, using a qualitative approach, various practices of television reception thematically consistent at group level were identified through social discourses of students from various schools of Punta Arenas, despite maintaining different dynamics at an individual level. These are the structuring of a daily routine that determines the interaction with television, the conviction of the students on the influence of media, its relationship with social institutions, the preference of certain television contents, the dynamics and ways of watching TV and the existence of a critical speech towards television programming.

In this way, as other investigations have agreed that television helped limit the hegemony of school as the only means to access information, this study also concluded that the school remains at the top of social interactions of the students. In this context, and noting the significance in the transformation of the schools that may or are have already been provoked by the information technologies, further work is required in order to consider the investigations around the interaction of various social groups- children, adolescents, adults, elderly, etc..., with these displays, not only in the school context, but in daily routines.

KEY WORDS: Television, Receptiveness, practices, students.

\section{INTRODUCCIÓN}

A pesar de las nuevas tecnologías audiovisuales surgidas en la actualidad y que parecen sustituir a los medios de comunicación tradicionales, la televisión, abierta o por cable, sigue siendo en América Latina el fenómeno cultural y mediático mayoritario (Orozco 2006). Algunos estudios internacionales incluso han establecido que el acceso de sectores amplios de la población a la computación e Internet, más que alejar a la televisión de las prácticas sociales cotidianas, ha fortalecido y complementado su capacidad para que ellas se inserten en nuevos contextos tecnológicos ${ }^{1}$. En este ámbito, los jóvenes han sido identificados como el grupo social que, por sus distintas formas de

1 Un estudio realizado en mayo del 2008 por el servicio de medios Nielsen en Estados Unidos y que encuestó a 3000 personas en más de 1000 hogares, arrojó como resultados un aumento de la dedicación de horas a la televisión en las personas que navegan por la red. consumo audiovisual, mantienen una mayor capacidad de socialización tecnológica, derivada principalmente por su trayectoria y vínculos con una cultura y sistema constituido por símbolos, aún cuando, en comparación con la infancia, disminuye notablemente el tiempo de exposición al televisor y comienza a ocurrir un doble desplazamiento hacia una mayor vida social y otras actividades de ocio (Lutczak 2006).

La indagación en las experiencias de socialización, relaciones y actividades de los grupos sociales en el contexto de la globalización y las convergencias tecnológicas, no sólo fundamenta el análisis de fenómenos comunicativos, sino permite entender la forma en que se construyen los procesos socio - históricos. Por este motivo, diversos estudios, entre los que se pueden mencionar los significativos aportes teóricos de Martín-Barbero y Guillermo Orozco al tema, señalan en sus conclusiones la necesidad de explorar en las prácticas de recepción mediática de los diversos sujetos sociales como una forma de contribuir al campo de 
la educación, partiendo de la premisa que la escuela no constituye el único medio por el cual las nuevas generaciones entran en contacto con el conocimiento y la información (Brunner 2000).

Los acelerados cambios políticos y económicos experimentados en el mundo en las dos últimas décadas del siglo XX, así como las sorprendentes evoluciones de los contextos tecnológicos fueron algunos de los factores que incidieron en la implementación de determinadas políticas públicas generadas en el marco de la Reforma Educacional impulsada por el Gobierno de Chile desde el año 1990.

En sus propuestas para el mejoramiento de la educación en Chile en el siglo XXI, el informe presentado por el Comité Técnico Asesor del Diálogo Nacional Sobre la Modernización de la Educación Chilena(1996), afirma que el aprendizaje no es un producto exclusivo de la sala de clases, sino que intervienen en él diversos factores socioculturales que pueden potenciarle o restarle efectividad, tales como la familia y el ambiente del hogar, el vecindario, los amigos y los medios de comunicación de masas, principalmente la televisión, que los niños empiezan a ver antes de asistir a la escuela. En relación a esto señala: Se han convertido en una poderosa agencia de socialización de los niños y los jóvenes, proporcionando información, entretención e, incluso, modelos de valor, uso del lenguaje, relaciones afectivas y estilos de consumo de vida. Sobre todo la televisión tiene la responsabilidad educacional de la familia y la escuela, y debe proteger a la infancia, eliminando de la pantalla - en los horarios de acceso a los niños- los elementos de violencia, sensacionalismo y truculencia que puedan distorsionar el desarrollo intelectual y afectivo en una etapa clave de la formación de su persona. La escuela, dentro de sus posibilidades, debiera tomar en cuenta este tipo de cultura audiovisual y su influencia concurrente sobre la formación de los alumnos.

En el Informe elaborado por el Consejo Asesor Presidencial para el mejoramiento de la Educación, la Presidenta de la República, Michelle Bachelet, señaló que la escuela debe ser también una puerta de acceso a los diversos medios por los que se crea, transmite y reproduce cultura ${ }^{2}$. Con la intención de articular el esfuerzo de instituciones del ámbito público, la empresa privada y la sociedad civil, el Consejo Nacional de Televisión ha implementado el proyecto

2 Consejo Asesor Presidencial para el Mejoramiento de la Calidad de la Educación en Chile, 2006.
Novasur, el cual busca mejorar la calidad de los ambientes de aprendizaje de los establecimientos educacionales chilenos a través de tres componentes: una programación audiovisual ajustada al marco curricular de la reforma educacional chilena; un sistema de guías pedagógicas que orienta el uso en aula de dicha programación y una asistencia técnico-pedagógica a la implementación de este servicio educativo en las comunidades escolares. ${ }^{3}$

De igual forma, la UNESCO reafirma esta política al definir sus parámetros de lo que será una adecuada educación en comunicación, a través de enseñar y aprender sobre los medios de comunicación más que con esos medios; incorporar el análisis creativo y la producción creativa; desarrollarla tanto en el ámbito de la educación formal como no formal; y promover el espíritu de comunidad, responsabilidad social, así como la autonomía personal. ${ }^{4}$

Sin embargo, Joan Ferrés plantea que estos criterios, aceptados por la mayor parte de los profesionales de la enseñanza, no debieran restringirse a la elaboración y uso de contenidos audiovisuales con fines pedagógicos, sino que debieran considerar las dimensiones fundamentales en que se desarrolla la experiencia del consumo de medios (Ferrés 2003).

Como una forma de responder a la escasez de estudios regionales sobre la relación de grupos sociales específicos y sus vínculos con medios de comunicación, la presente investigación indagó en las prácticas de recepción televisiva que sostienen los estudiantes de enseñanza media de Punta Arenas, principalmente desde las experiencias de consumo descritas en sus discursos sociales. Orientándose desde los ámbitos y situaciones variadas en que los investigadores latinoamericanos han abordado los estudios de recepción, se estableció, desde los aportes teóricos de Martín-Barbero $(1990,2003)$ y Orozco (1996), el presupuesto epistemológico de que los procesos de recepción rebasan la mera exposición $y$, por lo tanto, no están circunscritos al momento específico de vinculación directa entre los sujetos receptores y los referentes mediáticos, sino que transcurren a lo largo de varios escenarios, en los que a su vez se intercambian, producen y reproducen nuevas apropiaciones mediáticas. De igual forma, Maritza López de La Roche (2005), aludiendo al concepto

Página oficial Consejo Nacional de Televisión www.cntv.cl. 4 UNESCO, Seminario Youth Media Education, 2002, España. 
de realidad que señala Giddens, afirma que "no debe asumirse meramente como una descripción de objetos, sino como una experiencia de estar en el mundo y de interactuar con los otros y con el entorno natural social". A esto, se suman Berger y Luckmann (1995), quienes a través de su noción de construcción social de la realidad proponen la "realidad" desde la experiencia de la gente en su rutina diaria y no desde los saberes transmitidos desde las instituciones sociales.

De esta forma, aplicando una metodología que ha caracterizado a este tipo de estudios en América Latina como es la cualitativa y considerando que el análisis de la recepción se orienta hacia la interpretación sensible de la experiencia fenomenológica de las audiencias y a relatar la experiencia frente a textos mediáticos y configuraciones pertinentes del contexto donde ocurre esa experiencia (Flores, 2006), se pudo conocer las prácticas individuales y grupales que desarrollan los estudiantes con la televisión, además del discurso social que construyen al explicitar sus motivaciones, preferencias programáticas, opiniones, y relevancia de diversos contextos sociales (especialmente escolares) en sus prácticas mediáticas.

\section{METODOLOGÍA}

Se realizaron 3 focus groups con estudiantes de Tercer Año de Enseñanza Media, cuyas edades fluctuaban entre los 16 y 17 años ${ }^{5}$ y que eran pertenecientes a diversos establecimientos educacionales de la comuna, los cuales, a su vez, representaban a los diversos sistemas de administración de la educación en Chile: subvencionada, particular subvencionada y la particular pagada ${ }^{6}$. Esta técnica permitió acce-

5 Según Jacquinot 1997 los medios, a través de sus elementos simbólicos, influyen sobre la habilidad para ejercer actividades intelectuales específicas y que se encuentran relacionadas por la edad, las aptitudes previas en cada una de estas facultades, la condición social, la tarea que se debe cumplir y otros. Por tal motivo, la edad promedio de los estudiantes al desarrollar estas capacidades fluctúa entre los 12 a 17 años.

6 Los focus groups se realizaron en dependencias de la Universidad de Magallanes con estudiantes del Colegio Miguel de Cervantes (Particular Pagado)/ Liceo Experimental (Particular Subvencionado) y Liceo Politécnico Cardenal Raúl Silva Henríquez (Subvencionado Municipal). Cada uno de los focus groups fue registrado en video y, posteriormente, transcrito el audio para el análisis de los datos. Este proceso permitió conocer e incorporar el contexto de cada una de las respuestas entregadas por los participantes, así como facilitar su nivel de comprensión. der a una visión más global, ya que por una parte, el investigador se introduce y observa el entorno grupal en que se desarrolla el estudiante, y por otro, mediante un diálogo horizontal se produce una interacción más íntima entre el entrevistador y el entrevistado, lo que hace posible obtener datos difíciles de conseguir por otras vías.

Para realizar la convocatoria, se solicitó la colaboración de intermediarios pertenecientes a los establecimientos educacionales antes mencionados. De esta forma, se acordó seleccionar una muestra proporcional a la cantidad de cursos de Tercer Año que existieran en el colegio. Por ejemplo, si un establecimiento educacional tiene "tres cursos de Tercer Año Medio", la muestra debiera ser de dos estudiantes por curso. Esta selección fue al azar, intentando establecer un equilibrio cuantitativo en el sexo de los participantes. Sin embargo, en relación al sistema de televisión, se rigió principalmente por estudiantes que contaran con televisión tradicional o conexión de cable. No así con sistemas satelitales o digitales, debido a que son considerados como dispositivos que cuentan con un particular sistema tecnológico. La masificación de la televisión por cable en diferentes sectores de Punta Arenas, obligó a no excluir este sistema de televisión en la muestra.

Las preguntas realizadas a los participantes durante las sesiones se regían por una pauta que establecía un orden desde temas generales a particulares. El esquema temático fue el siguiente: 1 .Importancia de la televisión en sus vidas 2.-Contexto en el que ven televisión 3.-Preferencias programáticas y motivaciones 4.-Interpretaciones de los contenidos mediáticos 5.- Interacción de los contenidos televisivos en otros escenarios 6.- Vinculación con otros medios de comunicación.

De igual forma, se generaron preguntas de acuerdo a la dinámica de la entrevista y la conversación, entendiendo la diversidad de actores participantes de la muestra. Una vez recolectados los datos, se procedió a la organización de esta información como una forma de preparar el proceso de análisis. Esto significó la revisión del material (cintas de video) y la transcripción de los datos verbales en textos escritos. La revisión de los datos obtenidos a través de los focus groups, obligó a permanentes lecturas y observaciones de los textos. A la luz de la revisión de los datos, se procedió a la identificación de unidades 
de significado, categorización y asignación de códigos a las categorías. Es necesario destacar que cuando la recolección de datos se realiza con la técnica del focus groups, la unidad de análisis es el grupo (lo que expresa y construye). Por lo tanto, se consideró como segmentos de contenidos las intervenciones de cada uno de los estudiantes que participaron en las entrevistas. A través de un proceso de comparación constante entre cada unidad de análisis, se otorgaron significados a los segmentos y se descubrieron categorías, las cuales fueron identificadas a través de un código específico. Este código correspondió a las iniciales de las palabras principales que constituyen la denominación de la categoría.

Cada categorización se realizó por grupo de entrevistados $y$, posteriormente, se unieron las categorías de acuerdo a sus similitudes y relaciones. Cada categoría fue descrita e interpretada en términos de su significado. A continuación, se comenzó a comparar categorías, de igual forma como se realizó con las unidades de análisis, se identificaron similitudes, diferencias y posibles vínculos entre ellas. Identificar temas implicó localizar los patrones que aparecieron de manera repetida entre las categorías. Los temas han sido las bases de las conclusiones que han surgido del análisis. Con base a la selección de los temas y el establecimiento de relaciones entre categorías se comenzó a interpretar los resultados y entender el fenómeno de estudio.

\section{RESULTADOS}

\section{Prácticas de recepción y discurso social}

Las prácticas de recepción televisiva son únicas e irreproducibles. Sin embargo, en la rutina cotidiana de los estudiantes, estas cuentan con similitudes que, a nivel individual y grupal, conforman un patrón de actitudes, usos y expresiones que confieren sentido a sus consumos y permite dimensionar su inserción en procesos más globales. En este ámbito, se manifiesta la influencia del medio externo en la construcción de sus discursos, principalmente orientados a buscar una legitimidad que no encuentran en la estructura social dominada principalmente por el sector adulto. Por tal motivo, sus opiniones respecto a los contenidos televisivos mantienen una tendencia crítica y negativa respecto al rol social del medio televisivo, pero que evidencia ciertas contradicciones con las preferencias manifestadas por determinados contenidos ${ }^{7}$.

\section{Fragmentación de las audiencias}

En la investigación se pudo establecer que el ingreso de sectores de la población de Punta Arenas al sistema de televisión por cable, ha permitido una mayor oferta de contenidos televisivos que satisfacen los gustos de audiencias específicas, generando una mayor segmentación del mercado. De acuerdo a lo señalado por Guillermo Orozco, este factor de fragmentación y disolución de los grandes medios generó una equivocada idea sobre la inutilidad de este tipo de investigaciones, sin considerar justamente que esta multifragmentada audienciación de las sociedades contemporáneas constituye uno de los mayores desafíos de la rigurosidad de este tipo de estudios (Orozco 1996) y que obligará a ampliar los campos de la investigación de los medios y los contenidos audiovisuales, además de plantear nuevas interrogantes sobre los límites de la televisión como objeto de estudio. En la investigación no se evidenciaron diferencias significativas en el capital escolar de los estudiantes que poseen conexión por cable con los que poseen televisión abierta, existiendo una capacidad de adaptarse a los contextos conversacionales vinculados a contenidos televisivos. Si bien el nivel socioeconómico es un factor que influye significativamente en el acceso a la televisión por cable, la elección de no contar con esta alternativa también es determinada por decisiones relacionadas a las creencias del grupo familiar.

\section{En el campo de la educación}

El estudio coincide en algunas de sus conclusiones con investigaciones nacionales e internacionales realizadas en torno al consumo televisivo de adolescentes. Un ejemplo es la descripción que realiza el estudio cuantitativo desarrollado por el Consejo Nacional de Televisión en conjunto con otros centros de investigación, el cual señala:

7 Un ejemplo es la crítica hacia el desmedro que sufren las regiones en los contenidos de los medios nacionales, así como el escaso aporte cultural que estos proporcionan a la audiencia. Sin embargo, al indagar en sus consumos televisivos, los estudiantes no manifiestan interés por los programas de contenidos regionales, ni los culturales. 
Los adolescentes constituyen un grupo especial y que no responden necesariamente a la lógica piramidal de los estratos socioeconómicos, sino más bien a intereses y temáticas específicas. Al mismo tiempo, ponen en duda ciertos mitos en torno a una juventud más bien irresponsable, frívola y poco comprometida. Si bien los adolescentes están abiertos a nuevas miradas y experiencias, mantienen valores familiares muy arraigados. Están concientes de estar construyendo su futuro y en ese sentido el colegio es de gran importancia para ellos. Son capaces de desarrollar formas de percepción en que los medios de comunicación -nuevos y antiguos- se consumen simultáneamente construyendo un menú de contenidos propios que combina porciones de programas televisivos con el chat del momento, la conversación telefónica y la música de la radio o el $C D$. Sienten que en los medios de comunicación hay escasos referentes para construir su personalidad y por tanto, exigen más de aquel medio que está más presente en sus vidas: la televisión ${ }^{8}$.

Si bien el presente estudio concuerda en que las instituciones sociales más afectadas por las transformaciones en los modos del circular del saber son la escuela y la familia (Martín Barbero 2003), también recalca que son justamente estas las que tiene una mayor participación en la interacción de los estudiantes con el medio televisivo. No obstante que la televisión es un medio que funciona al margen de la escuela, ello no significa que esta institución la haya excluido de sus habituales prácticas pedagógicas. Muchos de los temas de conversación de los estudiantes son originados por los contenidos televisivos (deportes, farándula, ficción, etc), a lo que se suman los esfuerzos de profesores por incorporar algunos de estos contenidos en los debates grupales. Además, el uso de recursos audiovisuales por parte de algunos profesores se ha posicionado como una estrategia de enseñanza cada vez más frecuente en la sala de clases sobre todo en materias y asignaturas específicas. Esto permite validar el argumento sobre la capacidad humana para procesar símbolos, al advertir que estos devienen en una fuerza productiva y que actúan como estructurantes del sistema social a nivel mundial, señalando la pérdida de centralidad del libro como eje de aprendizaje (Ibid).

Igualmente, la organización de los tiempos en la rutina cotidiana de los estudiantes de Punta
Arenas coincide con la clasificación propuesta por Oscar Lutszack en su estudio "La televisión en la organización del tiempo cotidiano de los adolescentes escolarizados", surgido de entrevistas a jóvenes de entre 16 a 18 años. Esta señala que existe un tiempo escolar y/o productivo, un tiempo doméstico y un tiempo libre, lo cual manifiesta una similitud en las prácticas cotidianas y televisivas de los estudiantes a nivel latinoamericano. Sin embargo, en el caso particular de los estudiantes de Punta Arenas, contiene rasgos singulares influenciados por las políticas educativas nacionales y las características del entorno regional. Si bien coinciden en que durante esta etapa se produce un alejamiento de la televisión y un incremento de otras actividades en el tiempo libre, existen diferencias en el tipo de prácticas realizadas ${ }^{9}$.

De esta forma, se pudo establecer una clasificación de las prácticas de recepción televisiva manifestada por los estudiantes, la cual se refleja en el siguiente esquema (Figura 1).

\section{Estructuración de la rutina cotidiana}

Existe consenso al afirmar que, en comparación a la infancia, durante la adolescencia disminuye significativamente la interacción con la televisión. Algunos de los factores que producen este alejamiento se vinculan a las actividades que comienzan a desarrollar los jóvenes durante su rutina cotidiana, tales como cumplir con las obligaciones escolares y domésticas; salir con los amigos, ir a fiestas, practicar deporte u otras actividades de ocio. Desde esta perspectiva, los estudiantes de Punta Arenas requieren organizar su tiempo para poder interactuar con la televisión, principalmente por las horas ocupadas en las obligaciones escolares, ya sean dentro y fuera del colegio ${ }^{10}$.

Por su parte, el tiempo doméstico incluye aquellas obligaciones del hogar que se realizan tras retornar del colegio. Es durante el horario de las comidas (desayuno, almuerzo, once y cena) cuando el estudiante tiene la oportunidad de interactuar con la

9 El estudio de Fuenzalida (1997) señala que durante el tiempo de ocio los estudiantes incrementan sus actividades hacia la lectura y el cine, situación que no se visualizó en los focus groups con los estudiantes del presente estudio.

10 Un promedio de siete horas diarias ocupa el estudiante de Punta Arenas durante la jornada escolar, sin incluir las obligaciones escolares fuera del colegio. 


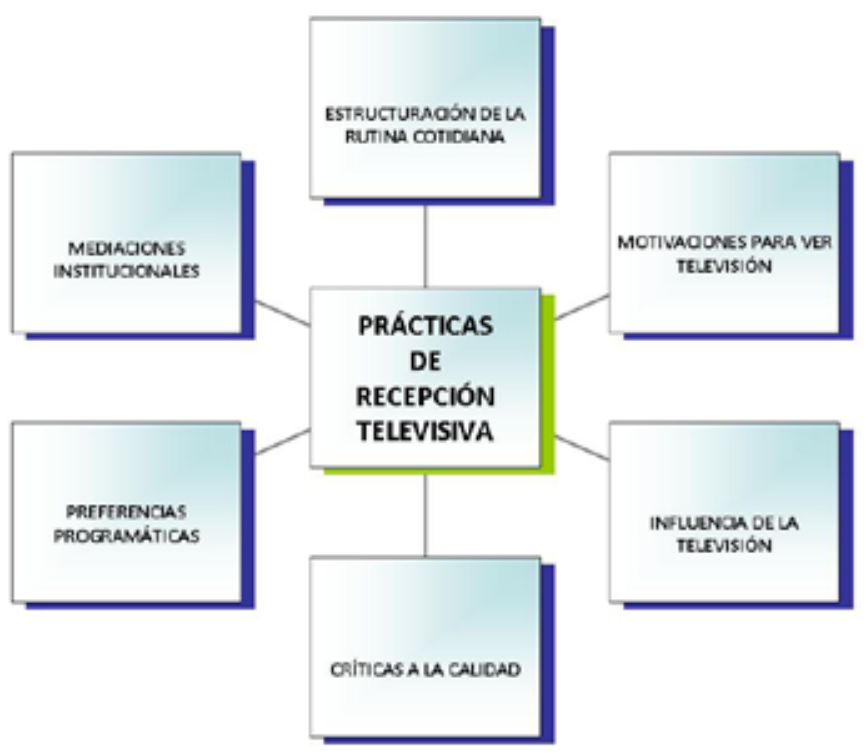

Fig. 1. Clasificación de prácticas de recepción televisiva.

televisión, lo cual ocurre generalmente acompañado por algún miembro del grupo familiar. El tiempo libre significa para los estudiantes el período que les permite realizar cualquier actividad ajena a las obligaciones escolares y domésticas. Se trata de un tiempo donde "no hay nada que hacer" y que puede ser dedicado a actividades de interés particular como salir con los amigos, hacer deportes, utilizar el computador o ver televisión.

\section{CONCLUSIONES}

\section{Las Motivaciones para ver televisión}

Las motivaciones que influyen en los estudiantes de Punta Arenas para interactuar con la televisión se encuentran asociadas a sus contextos particulares. Reconociendo en el contexto hogareño un espacio físico idóneo y estable para ver televisión, la motivación de Superar la rutina le otorga una significativa importancia a la escuela como institución social mediadora en la cotidianidad de los jóvenes: el "nada que hacer" muchas veces se traduce en cualquier actividad que no se vincule a la escuela. La estructuración de su rutina diaria y el tiempo ocupado en las actividades escolares, los obliga a contar con una selección de contenidos televisivos específicos. De esta forma, se motivan a través de la organización de su tiempo libre para disfrutarlos. La compañía familiar es otra de las motivaciones que suelen mencionar los estudiantes en su interacción con la televisión. Se suele dar durante el horario de las comidas, en la noche o por la predisposición familiar a presenciar un programa televisivo específico. Esta motivación se encuentra condicionada por diversos factores, tales como la decisión de los padres (la hora de ver televisión, restricción de contenidos, etc.), la ubicación del aparato televisivo e, incluso, la cantidad de televisores que existen al interior del hogar. Esto último no es menor, puesto que si bien estas prácticas son más habituales en grupos familiares que cuentan con un solo aparato receptor, esta motivación es valorada más significativamente en los estudiantes que cuentan con más de un televisor en su espacio hogareño ${ }^{11}$.

\section{La Televisión: Un medio influyente}

Si bien los estudiantes identifican a la televisión como un medio influyente en los gustos y conductas de las personas, sus argumentos dejan entrever juicios condicionados por la distancia generacional y trascendencia en el tiempo de las teorías sobre causa- efecto de los medios hacia las audiencias.

Reconocen en la infancia el período de mayor dedicación televisiva y a través de la comparación

11 El deseo de ver televisión acompañado de su familia corresponde para aquellos estudiantes que cuentan con un televisor en su dormitorio como un acto voluntario y excepcional. 
de los gustos infantiles actuales con las que ellos experimentaron durante esa etapa, afirman la influencia negativa que la televisión tiene sobre el comportamiento de los niños actuales. De igual forma, sostienen esta apreciación con el tema de la delincuencia, donde sindican a la televisión como un medio que genera estereotipos y modelos conductuales. Por una parte, acusan a la televisión y contenidos informativos de generar una "imagen negativa" de Santiago; por otra, de entregar a los delincuentes "tácticas" para cometer sus actos. En un plano más neutral, destacan la influencia significativa de la televisión en la publicidad de productos de consumo, reconociendo la permanencia de algunos spots comerciales en la memoria colectiva juvenil.

\section{La Construcción de un Discurso Crítico: buscando una legitimidad social}

El estudio permitió entrever la elaboración de un discurso crítico del estudiante hacia la televisión que, más allá de buscar una apreciación crítica y objetiva del fenómeno mediático, busca consolidar una posición de reconocimiento y legitimidad en la estructura social, donde priman significativamente los juicios del mundo adulto y sus líderes de opinión. Este se construye a partir de una constante mención de atributos negativos del medio televisivo, vinculados a crear un modelo carente de valores $y$ responsabilidad con el medio social. Sin embargo, surgen contradicciones en este discurso cuando se comparan sus opiniones críticas con las preferencias televisivas anteriormente mencionadas.

Entendiendo el concepto de cultura desde una perspectiva excluyente y vinculada al campo de las artes, las ciencias y la educación, los estudiantes cuestionan a la televisión su escaso aporte cultural. Esta afirmación permite observar que para los estudiantes la televisión no es considerada como representante y canalizadora de las diversas manifestaciones y expresiones del medio social. Los programas identificados como de farándula son cuestionados en su mayoría por los estudiantes, argumentando los valores negativos que promueve en la juventud. Sin embargo, afirman reconocer gran parte de sus contenidos, los cuales se convierten en temas de conversación entre sus compañeros del colegio. La crítica a los contenidos violentos de los dibujos animados actuales refuerza el reconocimiento que ellos tienen de la etapa infantil con la televisión. En este contexto, los dibujos animados de origen asiático - identificados por los estudiantes como "monos japoneses"- son, a su juicio, los principales promotores de esta violencia manifestada en escenas visuales "sangrientas" y un escaso cuestionamiento moral frente al acto de matar. Por último, y a pesar de la valoración positiva que sostienen en torno a los programas informativos, critican el tratamiento que los medios realizan de la información, argumentando un excesivo centralismo geográfico, el cual excluye las noticias de regiones apartadas como Magallanes; y la saturación que producen los noticieros en torno a un acontecimiento.

\section{Preferencias Programáticas: la} hegemonía de la ficción

Los estudiantes cuentan con preferencias programáticas del sistema de televisión tradicional y de cable, abarcando diversos formatos y géneros. En algunas situaciones y de acuerdo al contexto social, se manifiestan coincidencias por determinados contenidos principalmente vinculados a las producciones de ficción, tales como series, miniseries, telenovelas y películas. Existe una clara tendencia por las series norteamericanas de ficción, principalmente por aquellos estudiantes que cuentan con televisión por cable. Sin embargo, son las mujeres quienes reconocen ser seguidoras de series como Dr. House ${ }^{12}$, Desperate Housewifes, Grey's Anatomy, las cuales, afirman, vuelven a verlas cuando estas se exhiben por la televisión tradicional. La serie norteamericana Dr. House y Los Simpsons se encuentran entre sus preferencias mayoritarias.

La preferencia por largometrajes de ficción se caracteriza por una tendencia hacia las producciones industriales norteamericanas que se convirtieron en importantes éxitos de público a nivel mundial en el circuito cinematográfico; mientras que los programas deportivos, son preferidos en su mayoría por

12 Los estudiantes demuestran una empatía con las actitudes y personalidad del personaje, las cuales representan valores que se apartan del modelo tradicional de héroe clásico. $\mathrm{Al}$ contrario, el personaje del Dr. House es individualista irónico, narcisista. De acuerdo a su creador, el personaje se encuentra inspirado en Sherlock Holmes, sin embargo, este antecedente no fue mencionado por los estudiantes. 
los varones, principalmente por quienes practican algún deporte o diversifican sus preferencias en distintos formatos televisivos como series - reportajes; programas espectáculos, eventos deportivos masivos y canales dedicados a deportes específicos. De igual forma, y a pesar del discurso crítico sobre sus contenidos, existe una evidente opción de los estudiantes por los programas misceláneos juveniles y que se exhiben durante las tardes por la televisión tradicional.

Manifestando una tendencia a recordar aquellos acontecimientos vinculados a desastres naturales, accidentes o actos de violencia ${ }^{13}$, los estudiantes ven en los programas informativos verdaderos puentes de conexión entre la realidad individual y social, principalmente a través de programas o reportajes dedicados a la denuncia pública ${ }^{14}$. Las motivaciones por este tipo de contenidos pueden abarcar desde el interés por informarse, la compañía familiar o ser la mejor alternativa ante una programación que no despierta ningún otro interés.

Mediaciones Institucionales:

La Escuela y la Familia

Las mediaciones institucionales son aquellas estructuras incrustadas en las prácticas sociales que resultan de la participación del individuo en las diversas instituciones: familia, escuela, empresa, grupos de amigos o vecindad (Orozco 2004). De las conclusiones del estudio, la escuela y la familia son las dos instituciones sociales más importantes que intervienen en los procesos de interacción de los estudiantes con la televisión.

En el caso específico de la familia, su mediación se manifiesta en diversos contextos, entre los cuales se puede mencionar la influencia en las frecuencias y horarios que permiten la interacción televisiva del estudiante, la restricción en los horarios y contenidos televisivos; o la compañía familiar.

Cada grupo familiar cuenta con un ritual particular, el cual se encuentra condicionado por

13 Los atentados terroristas a Estados Unidos el año 2001 y la erupción del Volcán Chaitén son los hechos noticiosos más recordados a nivel internacional y nacional respectivamente.

14

Los estudiantes otorgan a la televisión un carácter mediador y representacional de la realidad, lo cual se evidencia en sus preferencias por los programas de denuncias que utilizan cámaras ocultas y que captan situaciones reñidas con la ley actividades diarias obligatorias. En el caso de los jóvenes, el cumplimiento de los deberes escolares; en el caso de los padres, las actividades laborales o domésticas. Cuando el grupo familiar se reúne en horario nocturno para presenciar un contenido televisivo, esta situación acontece frente al interés por programas como misceláneos, concursos, películas.

Los jóvenes reconocen la importancia de la familia en el hecho de ver las noticias, principalmente por la situación particular de compartir un tiempo específico - "el horario de noticias"- o bien, porque forma parte del interés y motivación común. Sin embargo, también compartir la televisión con la familia es parte de un ritual que se encuentra condicionado a las normas y disposiciones de algún integrante o el grupo en general.

Considerando su propia situación como jóvenes insertos en el sistema educativo, la mediación de la institución escolar se realiza, en primer lugar, a través de la interacción con los compañeros del colegio, y en segundo lugar, con los profesores. En ambos casos, la conversación sobre algún tema puntual vinculado a la televisión (programas, series, películas, noticias, etc.) se desarrolla tanto fuera como dentro de la sala de clases. Además, los temas de conversación varían considerablemente entre los diversos actores participantes del proceso de mediación escolar. Mientras con el profesor se genera en un contexto pedagógico, con los compañeros del colegio acontece en temas relacionados a sus intereses particulares como jóvenes. Entre estos se puede mencionar las conversaciones sobre deportes (más habitual en estudiantes varones); las situaciones humorísticas o polémicas que ocurren en ciertos programas; o alguna película de ficción ${ }^{15}$.

A diferencia de la mediación familiar, el espacio físico del colegio no se convierte en el lugar propicio para interactuar con la televisión, la cual encuentra su posible analogía tan solo en el uso pedagógico de recursos audiovisuales que realiza el profesor al interior del aula. Esta estrategia de enseñanza, positivamente evaluada por los estudiantes, se caracteriza por la utilización que realiza el profesor, de películas

15 Uno de los grupos de estudiantes se refirió a la importancia del filme "Titanic" como tema de conversación en el colegio, lo cual permite suponer la existencia de referentes cinematográficos que permanecen en la imaginería colectiva generacional y que se refuerzan cuando estos son exhibidas por las pantallas de la televisión. 
o documentales como material de apoyo ${ }^{16}$, lo cual le otorga mayor dinamismo a las clases, posibilidad de generar opiniones y evitar - o al menos atenuar- el agotador acto de escribir. La efectividad de estos recursos pedagógicos innovadores en la sala de clases, que se está haciendo cada vez más habitual, tiene una relación de causa y efecto en las motivaciones cognitivas del estudiante. Por una parte, establece una mediación entre producción y recepción de contenidos, posibilitando al estudiante incorporar estas informaciones a sus prácticas académicas y culturales ${ }^{17}$.

\section{BIBLIOGRAFÍA}

BERGER, P. y T. LUCKMANN 1995 [1968]. La Construcción Social de la realidad. Amorrortu editores, Buenos Aires.

BRUNNER, J.J. 2000. Nuevos escenarios de la educación. Revolución tecnológica y Sociedad de la Información. PREAL, Santiago.

CNTV. 2007. MacCann-Erickson y Research Chile.

DURKHEIM, E. 1951 [1898] Representaciones individuales y representaciones colectivas. En Sociología y Filosofía. Editorial Guillermo Kraft, Buenos Aires.

FERRÉS, J. 2003. Educación en medios y competencia emocional. Revista Iberoamericana de Educación 32:49-69.

FLORES, C. 2006. El análisis de la recepción: estudio cualitativo de audiencias en TV. Boletín Datos, Diagnósticos y Tendencias julio-septiembre: s/pp.

FUENZALIDA, V. 1997. Televisión y cultura cotidiana. Corporación de Promoción Universitaria, Santiago.

FUENZALIDA, V. y M.E. HERMOSILLA 1992. El televidente activo. Corporación de Promoción Universitaria, Santiago.

GARCIA-CANCLINl, N. 1989. Culturas Híbridas. Grijalbo/ FNCA, México.

GIDDENS, A. 1991. Modernity and Self-Identity. Stanford University Press, Stanford.

2006. Informe del Consejo Asesor Presidencial para el Mejoramiento de la Calidad de la Educación en Chile. MS.

24 De acuerdo a los testimonios de los estudiantes son los profesores de Castellano e Historia, quienes suelen utilizar permanentemente este tipo de recursos, principalmente a través de películas y documentales. Le siguen Ciencias naturales y Química.

25 Este tipo de recursos y estrategias pedagógicas, abre interrogantes sobre los niveles de mediación que ejercen los contenidos audiovisuales respecto a sus objetivos curriculares, si consideramos que los estudiantes pueden asumir análogamente los contenidos de un texto literario o un hecho histórico con la códigos del lenguaje audiovisual o la síntesis y libertad de adaptación que se permite la ficción cinematográfica. Un ejemplo es la utilización de la película "300" para explicar el tema de los espartanos.
JACQUINOT, G. 1997. La Escuela frente a las pantallas. Aique, Buenos Aires.

JENSEN, K. 1992. El análisis de la recepción: la comunicación de masas como producción social de significado. En: Metodologías cualitativas de investigación en comunicación de masas. Jensen, K. y Jankowski, N. editores. pp. 165-180. Bosch, Barcelona.

LÓPEZ DE LA ROCHE, M. Y MÉNDEZ ÁLVAREZ, L. 2005 Representaciones Sociales construidas por audiencias infantiles, a partir del análisis de las Mediaciones ejercidas por los medios y tecnologías de la comunicación. Revista Colombiana de Educación 46:156-185.

LOZANO, J. 2006. Televisión y vida cotidiana en México, Guadalajara y Monterrey: marco de referencia para el trabajo de campo (encuestas, entrevistas y grupos de discusión). Cátedra Televisa, Instituto Tecnológico y de Estudios Superiores de Monterrey.

LUTCZAK, O. 2006. La Televisión en la organización del tiempo cotidiano de los adolescentes escolarizados. En: ¿Y la recepción? Balance crítico de los estudios sobre el público. Saintout, F. y N. Ferrante, editoras. La Crujía Ediciones, Buenos Aires.

MARTÍN-BARBERO, J. 1990. De los medios a las mediaciones. Gustavo Gili, México.

2003. Saberes hoy, diseminaciones, competencias y transversalidades, Revista Iberoamericana de Educación 32: $17-34$.

MAZZIOTTI, N. 2006. Estudios sobre Recepción, una exploración constante. En: ¿Y la recepción? Balance crítico de los estudios sobre el público. Saintout, F. y N. Ferrante, editoras. La Crujía Ediciones, Buenos Aires.

OROZCO, G. 1996. Televisión y audiencias: un enfoque cualitativo. Proyecto Didáctico Quirón. Editorial de la Torre, Universidad Iberoamericana, Madrid.

2006 Los Estudios de Recepción: De un modo de investigar a una moda y de ahí a muchos modos. En: ¿Y la recepción? Balance crítico de los estudios sobre el público. Saintout, F. y N. Ferrante, editoras. La Crujía Ediciones, Buenos Aires.

PEYRÚ, G. 1993. Papá, ¿puedo ver la tele? Paidós, Buenos Aires. SAINTOUT, F y N. FERRANTE 2004. ¿Y la Recepción? Balance crítico de los estudios sobre el público. Libro de compilaciones de Argentina, Buenos Aires.

VASALLO DE LOPES, M. I. 2003. Reflexiones teóricometodológicas dentro de un estudio de recepción. Tra(m)pas de la Comunicación y la Cultura, La Plata, Argentina, 1(12):s/pp.

VEGA, A. 2005. Construyendo puentes: La identidad de género de los jefes de familia y la recepción televisiva. Comunicación y Sociedad 4:127-145. 